\title{
Representing Constraints with Automata
}

\author{
Frank Morawietz and Tom Cornell \\ Seminar für Sprachwissenschaft \\ Universität Tübingen \\ Wilhelmstr. 113 \\ 72074 Tübingen, Germany \\ \{frank, cornell\}@sfs .nphil.uni-tuebingen.de
}

\begin{abstract}
In this paper we describe an approach to constraint based syntactic theories in terms of finite tree automata. The solutions to constraints expressed in weak monadic second order (MSO) logic are represented by tree automata recognizing the assignments which make the formulas true. We show that this allows an efficient representation of knowledge about the content of constraints which can be used as a practical tool for grammatical theory verification. We achieve this by using the intertranslatability of formulae of MSO logic and tree automata and the embedding of MSO logic into a constraint logic programming scheme. The usefulness of the approach is discussed with examples from the realm of Principles-and-Parameters based parsing.
\end{abstract}

\section{Introduction}

In recent years there has been a continuing interest in computational linguistics in both model theoretic syntax and finite state techniques. In this paper we attempt to bridge the gap between the two by exploiting an old result in logic, that the weak monadic second order (MSO) theory of two successor functions (WS2S) is decidable (Thatcher and Wright 1968, Doner 1970). A "weak" second order theory is one in which the set variables are allowed to range only over finite sets. There is a more powerful result available: it has been shown (Rabin 1969) that the strong monadic second order theory (variables range over infinite sets) of even countably many successor functions is decidable. However, in our linguistic applications we only need to quantify over finite sets, so the weaker theory is enough, and the techniques cor- respondingly simpler. ${ }^{1}$ The decidability proof works by showing a correspondence between formulas in the language of WS2S and tree automata, developed in such a way that the formula is satisfiable iff the set of trees accepted by the corresponding automaton is nonempty. While these results were well known, the (rather surprising) suitability of this formalism as a constraint language for Principles and Parameters (P\&P) based linguistic theories has only recently been shown by Rogers (1994).

It should be pointed out immediately that the translation from formulas to automata, while effective, is just about as complex as it is possible to be. In the worst case, the number of states can be given as a function of the number of variables in the input formula with a stack of exponents as tall as the number of quantifier alternations in the formula. However, there is a growing body of work in the computer science literature motivated by the success of the MONA decision procedure (Henriksen et al. 1995) $)^{2}$ on the application of these techniques in computer science (Basin and Klarlund 1995, Kelb et al. 1997), which suggests that in practical cases the extreme explosiveness of this technique can be effectively controlled. It is one of our goals to show that this is the case in linguistic applications as well.

The decidability proof for WS2S is inductive on the structure of MSO formulas. Therefore we can choose our particular tree description language rather freely, knowing (a) that the resulting logic

\footnotetext{
${ }^{1}$ All of these are generalizations to trees of results on strings and the monadic second order theory of one successor function originally due to Büchi (1960). The applications we mention here could be adapted to strings with finite-state automata replacing tree automata. In general, all the techniques which apply to tree automata are straightforward generalizations of techniques for FSAs.

${ }^{2}$ The current version of the MONA tool works only on the MSO logic of strings. There is work in progress at the University of Aarhus to extend MONA to "MONA++", for trees (Biehl et al. 1996).
} 
will be decidable and (b) that the translation to automata will go through as long as the atomic formulas of the language represent relations which can be translated (by hand if necessary) to tree automata. We will see how this is done in the next section, but the point can be appreciated immediately. For example, Niehren and Podelski (1992) and Ayari et al. (1997) have investigated the usefulness of these techniques in dealing with feature trees which unfold feature structures; there the attributes of an attribute-value term are translated to distinct successor functions. On the other hand, Rogers (1996) has developed a language rich in long-distance relations (dominance and precedence) which is more appropriate for work in Government-Binding (GB) theory. Compact automata can be easily constructed to represent dominance and precedence relations. One can imagine other possibilities as well: as we will see, the automaton for Kayne-style asymmetric, precedence-restricted c-command (Kayne 1994) is also very compact, and makes a suitable primitive for a description language along the lines developed by Frank and Vijay-Shanker (1995).

The paper is organized as follows. First we present some of the mathematical background, then we discuss (naïve) uses of the techniques, followed by the presentation of a constraint logic programmingbased extension of MSO logic to avoid some of the problems of the naive approach, concluding with a discussion of its strengths and weaknesses.

\section{Defining Automata with Constraints}

Tree automata. For completeness, we sketch the definitions of trees and tree automata here. An introduction to tree automata can be found in Gécseg and Steinby (1984), as well as in Thatcher and Wright (1968) and Doner (1970).

Assume an alphabet $\Sigma=\Sigma_{0} \cup \Sigma_{2}$ with $\Sigma_{0}=\{\lambda\}$ and $\Sigma_{2}$ being a set of binary operation symbols. We think of (binary) trees over $\Sigma$ as just the set of terms $T_{\Sigma}$ constructed from this alphabet. That is, we let $\lambda$ be the empty tree and let $\sigma\left(t_{1}, t_{2}\right)$, for $\sigma \in \Sigma_{2}$ and $t_{1}, t_{2} \in T_{\Sigma}$, denote the tree with label $\sigma$ and subtrees $t_{1}, t_{2}$. Alternatively, we can think of a tree $t$ as a function from the addresses in a binary tree domain $T$ to labels in $\Sigma .^{3}$

A deterministic (bottom-up) tree automaton $\mathcal{A}$ on binary trees is a tuple $\left\langle A, \Sigma, a_{0}, F, \alpha\right\rangle$ with $A$ the set

\footnotetext{
${ }^{3}$ The first approach is developed in Thatcher and Wright (1968), the second in Doner (1970). A tree domain is a subset of strings over a linearly ordered set which is closed under prefix and left sister.
}

of states, $a_{0} \in A$ the initial state, $F \subseteq A$ the final states and $\alpha:(A \times A \times \Sigma) \rightarrow A$ the transition function. The transition function can be thought of as a homomorphism on trees inductively defined as: $h_{\alpha}(\lambda)=a_{0}$ and $h_{\alpha}\left(\sigma\left(t_{1}, t_{2}\right)\right)=\alpha\left(h_{\alpha}\left(t_{1}\right), h_{\alpha}\left(t_{2}\right), \sigma\right)$. An automaton $\mathcal{A}$ accepts a tree $t$ iff $h_{\alpha}(t) \in F$. The language recognized by $\mathcal{A}$ is denoted by $T(\mathcal{A})=$ $\left\{t \mid h_{\alpha}(t) \in F\right\}$.

Emptiness of the language $T(\mathcal{A})$ is decidable by a fixpoint construction computing the set of reachable states. The reachability algorithm is given below in Figure 1. $R$ contains the reachable states constructed so far, and $R^{\prime}$ contains possibly new states constructed on the current pass through the loop. $T(\mathcal{A})$ is empty if and only if no final state is reach-

1. $R:=\left\{a_{0}\right\}, R^{\prime}:=\emptyset$.

2. For all $\left(a_{i}, a_{j}\right) \in R \times R$, for all $\sigma \in \Sigma$, $R^{\prime}:=R^{\prime} \cup\left\{\alpha\left(a_{i}, a_{j}, \sigma\right)\right\}$.

3. If $R^{\prime}-R=\emptyset$ then return $R$, else $R:=R \cup R^{\prime}$, go to step 2 .

Figure 1: Reachable states algorithm.

able. Naturally, if we want to test emptiness, we can stop the construction as soon as we encounter a final state in $R^{\prime}$. Note that, given an automaton with $k$ states, the algorithm must terminate after at most $k$ passes through the loop, so the algorithm terminates after at most $k^{3}$ searches through the transition table.

Sets of trees which are the language of some tree automaton are called recognizable. ${ }^{4}$ The recognizable sets are closed under the boolean operations of conjunction, disjunction and negation, and the automaton constructions which witness these closure results are absolutely straightforward generalizations of the corresponding better-known constructions for finite state automata. The recognizable sets are also closed under projections (mappings from one alphabet to another) and inverse projections, and again the construction is essentially that for finite state automata. The projection construction yields a nondeterministic automaton, but, again as for FSA's, bottom-up tree automata can be made deterministic by a straightforward generalization of the subset construction. (Note that top-down tree automata do not have this property: deterministic top-down tree automata recognize a strictly narrower family of tree sets.) Finally, tree automata can

\footnotetext{
${ }^{4}$ The recognizable sets of trees yield the context free string languages, so MSO logics are limited to context free power. However, the CLP extension discussed below can be used to amplify the power of the formalism where necessary.
} 
be minimized by a construction which is, yet again, a straightforward generalization of well known FSA techniques.

The weak second order theory of two successor functions. One attraction of monadic second order tree logics is that they give us a principled means of generating automata from a constraintbased theory. The connection allows the linguist to specify ideas about natural language in a concise manner in logic, while at the same time providing a way of "compiling" those constraints into a form which can be efficiently used in natural language processing applications.

The translation is provided via the weak monadic second order theory of two successor functions (WS2S). The structure of two successor functions, $\mathcal{N}_{2}$, has for its domain $\left(N_{2}\right)$ the infinite binary branching tree. Standardly the language of WS2S is based on two successor functions (left-daughter and right-daughter), but, as Rogers (1994) shows, this is intertranslatable with a language based on dominance and precedence relations. Because we choose the monadic second order language over whichever of these two signatures is preferred, we can quantify over sets of nodes in $N_{2}$. So we can use these sets to pick out arbitrarily large finite trees embedded in $N_{2}$. Second order variables can also be used to pick out other properties of nodes, such as category or other node-labeling features, and they can be used to pick out higher order substructures such as $\overline{\mathrm{X}}$ projections or chains.

As usual, satisfiability of a formula in the language of WS2S by $\mathcal{N}_{2}$ is relative to an assignment function, mapping individual variables to members of $N_{2}$ (as in first order logic) and mapping monadic predicate variables to subsets of $N_{2}$. Following Büchi (1960), Doner (1970) and Thatcher and Wright (1968) show that assignment functions for such formulas can be coded by a labeling of the nodes in $N_{2}$ in the following way. First, we treat individual variables as set variables which are constrained to be singleton sets (we can define the singletonhood property in MSO tree logic). So, without loss of generality, we can think of the domain of the assignment function as a sequence $X_{1}, \ldots, X_{n}$ of the variables occurring in the given formula. We choose our labeling alphabet to be the set of length $n$ bit strings: $\{0,1\}^{n}$. Then, for every node $n \in N_{2}$, if we intend to assign $n$ to the denotation of $X_{i}$, we indicate this by labeling $n$ with a bit string in which the $i$ th bit is on. (In effect, we are labelling every node with a list of the sets to which it belongs.) Now every assignment function we might need corresponds uniquely to a labeling function over $N_{2}$. What Doner, and Thatcher and Wright (and, for strong S2S, Rabin) show is that each formula in the language of WS2S corresponds to a tree automaton which recognizes just the satisfying "assignment labelings", and we can thereby define a notion of "recognizable relation". So the formula is satisfiable just in case the corresponding automaton recognizes a nonempty language. Note that any language whose formulas can be converted to automata in this way is therefore guaranteed to be decidable, though whether it is as strong as the language of WS2S must still be shown.

This approach to theorem-proving is rather different from more general techniques for higher-order theorem proving in ways that the formalizer must keep in mind. In particular, we are deciding membership in the theory of a fixed structure, $\mathcal{N}_{2}$, and not consequence of an explicit set of tree axioms. So, for example, the parse tree shows up in the formalization as a second order variable, rather than simply being a satisfying model (cf. Johnson (1994), on "satisfiability-based" grammar formalisms).

As an example consider the following formula denoting the relation of directed asymmetric ccommand $^{5}$ in the sense of Kayne (1994). We use the tree logic signature of Rogers (1994), which, in a second order setting, is interdefinable with the language of multiple successor functions. Uppercase letters denote second order variables, lowercase ones first order variables, $\triangleleft^{*}$ reflexive domination, $\triangleleft^{+}$proper domination and $\prec$ proper precedence:

$$
\begin{aligned}
& A C-\operatorname{Com}\left(x_{1}, x_{2}\right) \stackrel{\text { def }}{\Longleftrightarrow} \\
& \% x \text { c-commands } y: \\
& (\forall z)\left[z \triangleleft^{+} x \Rightarrow z \triangleleft^{+} y\right] \wedge \neg\left(x \triangleleft^{*} y\right) \wedge \\
& \% y \text { does not c-command } x: \\
& \neg\left((\forall z)\left[z \triangleleft^{+} y \Rightarrow z \triangleleft^{+} x\right] \wedge \neg\left(y \triangleleft^{*} x\right)\right) \wedge \\
& \% x \text { preceeds } y: \\
& x \prec y
\end{aligned}
$$

The corresponding tree automaton is shown in Figure 2. On closer examination of the transitions, we note that we just percolate the initial state as long as we find only nodes which are neither $x_{1}$ nor $x_{2}$. From the initial state on both the left and the right subtree we can either go to the state denoting "found $x_{1}$ " $\left(a_{1}\right)$ if we read symbol $\underline{10}$ or to the state denoting "found $x_{2}$ " $\left(a_{2}\right)$ if we read symbol $\underline{01}$. We can then percolate $a_{2}$ as long as the other branch does not immediately dominate $x_{1}$. When we have

\footnotetext{
${ }^{5}$ This relation is not monadic, but reducible via syntactic substitution to an MSO signature. In fact, we can define relations of any arity as long as they are explicitly presentable in MSO logic.
} 


$$
\begin{gathered}
\mathcal{A}=\left\langle A, \Sigma, a_{0}, F, \alpha\right\rangle, \\
A=\left\{a_{0}, a_{1}, a_{2}, a_{3}, a_{4}\right\}, \\
\Sigma=\{\underline{11}, \underline{10}, \underline{01}, \underline{00}\} \\
F=\left\{a_{3}\right\}
\end{gathered}
$$

$$
\begin{array}{ll}
\alpha\left(a_{0}, a_{0}, \underline{00}\right)=a_{0} & \alpha\left(a_{0}, a_{0}, \underline{10}\right)=a_{1} \\
\alpha\left(a_{0}, a_{0}, \underline{01}\right)=a_{2} & \alpha\left(a_{0}, a_{2}, \underline{\underline{00}}\right)=a_{2} \\
\alpha\left(a_{0}, a_{3}, \underline{\underline{00}}\right)=a_{3} \quad \alpha\left(a_{2}, a_{0}, \underline{\underline{00}}\right)=a_{2} \\
\alpha\left(a_{1}, a_{2}, \underline{00}\right)=a_{3} \quad \alpha\left(a_{3}, a_{0}, \underline{00}\right)=a_{3} \\
\text { all other transitions are to } a_{4}
\end{array}
$$

Figure 2: The automaton for $A C$ - $\operatorname{Com}\left(x_{1}, x_{2}\right)$

$a_{1}$ on the left subtree and $a_{2}$ on the right one, we go to the final state $a_{3}$ which again can be percolated as long as empty symbols are read. Clearly, the automaton recognizes all trees which have the desired c-command relation between the two nodes. It compactly represents the (infinite) number of possible satisfying assignments.

The proof of the decidability of WS2S furnishes a technique for deriving such automata for recognizable relations effectively. (In fact the above automaton was constructed by a simple implementation of such a compiler which we have running at the University of Tübingen. See Morawietz and Cornell (1997).) The proof is inductive. In the base case, relations defined by atomic formulas are shown to be recognizable by brute force. Then the induction is based on the closure properties of the recognizable sets, so that logical operators correspond to automaton constructions in the following way: conjunction and negation just use the obvious corresponding automaton operations and existential quantification is implemented with the projection construction. The inductive nature of the proof allows us a fairly free choice of signature, as long as our atomic relations are recognizable. We could, for example, investigate theories in which asymmetric c-command was the only primitive, or asymmetric c-command plus dominance, for example.

The projection construction, as noted above, yields nondeterministic automata as output, and the negation construction requires deterministic automata as input, so the subset construction must be used every time a negated existential quantifier is encountered. The corresponding exponential blowup in the state space is the main cause of the nonelementary complexity of the construction. Since a quantifier prefix of the form $\exists \cdots \exists \forall \cdots \forall \exists \cdots$ is equivalent to $\exists \cdots \exists \neg \exists \cdots \exists \neg \exists \cdots$ we see that the stack of exponents involved is determined by the number of quantifier alternations.
It is obviously desirable to keep the automata as small as possible. In our own prototype, we minimize the outputs of all of our automata constructions. Note that this gives us another way of determining satisfiability, since the minimal automaton recognizing the empty language is readily detectable: its only state is the initial state, and it is not final.

\section{Defining Constraints with Automata}

An obvious goal for the use of the discussed approach would be the (offline) generation of a tree automaton representing an entire grammar. That is, in principle, if we can formalize a grammar in an MSO tree logic, we can apply these compilation techniques to construct an automaton which recognizes all and only the valid parse trees. ${ }^{6}$ In this setting, the parsing problem becomes the problem of conjoining an automaton recognizing the input with the grammar automaton, with the result being an automaton which recognizes all and only the valid parse trees. For example, assume that we have an automaton $\operatorname{Gram}(X)$ such that $X$ is a well-formed tree, and suppose we want to recognize the input John sees Mary. Then we conjoin a description of the input with the grammar automaton as given below.

$$
\begin{aligned}
& (\exists x, y, z \in X)[x \in \operatorname{John} \wedge y \in \operatorname{Sees} \wedge z \in \operatorname{Mary} \wedge \\
& \quad x \prec y \prec z \wedge \operatorname{Gram}(X)]
\end{aligned}
$$

The recognition problem is just the problem of determining whether or not the resulting automaton recognizes a nonempty language. Since the automaton represents the parse forest, we can run it to generate parse trees for this particular input.

Unfortunately, as we have already noted, the problem of generating a tree automaton from an arbitrary MSO formula is of non-elementary complexity. Therefore, it seems unlikely that a formalization of a realistic principle-based grammar could be compiled into a tree automaton before the heat death of the universe. (The formalization of ideas from Relativized Minimality (Rizzi 1990) presented in Rogers (1994) fills an entire chapter without specifying even the beginning of a full lexicon, for example.) Nonetheless there are a number of ways in which these compilation techniques remain useful. First, though the construction of a grammar automaton is almost certainly infeasible for realistic grammars, the construction of a grammar-andinput automaton-which is a very much smaller

\footnotetext{
${ }^{6}$ This is reminiscent of approaches associated with Bernard Lang. See van Noord (1995) and references therein.
} 
machine-may not be. We discuss techniques based on constraint logic programming that are applicable to that problem in the next section.

Another use for such a compiler is suggested by the standard divide-and-conquer strategy for problem solving: instead of compiling an entire grammar formula, we isolate interesting subformulas, and attempt to compile them. Tree automata represent properties of trees and there are many such properties less complex than global well-formedness which are nonetheless important to establish for parse trees. In particular, where the definition of a property of parse trees involves negation or quantification, including quantification over sets of nodes, it may be easier to express this in an MSO tree logic, compile the resulting formula, and use the resulting automaton as a filter on parse trees originally generated by other means (e.g., by a covering phrase structure grammar).

At the moment, at least, the question of which grammatical properties can be compiled in a reasonable time is largely empirical. It is made even more difficult by the lack of high quality software tools. This situation should be alleviated in the near future when work on MONA++ at the University of Aarhus is completed; the usefulness of its older sister MONA (Henriksen et al. 1995), which works on strings and FSA's, has been well demonstrated in the computer science literature. In the meantime, for tests, we are using a comparatively simple implementation of our own. Even with very low-power tools, however, we can construct automata for interesting grammatical constraints.

For example, recall the definition of asymmetric ccommand and its associated automaton in Figure 2. In linguistic applications, we generally use versions of c-command which are restricted to be local, in the sense that no element of a certain type is allowed to intervene. The general form of such a locality condition $L C$ might then be formalized as follows.

$$
\begin{aligned}
& L C(x, y) \stackrel{\text { def }}{\Longleftrightarrow} \\
& A C-C o m m(x, y) \wedge \\
& \% \text { there does not exist } z \text { with property } P: \\
& (\neg \exists z)[z \in P \wedge \\
& \quad \% \text { such that it intervenes between } x \text { and } y: \\
& \left.(\exists w)\left[w \triangleleft x \wedge w \triangleleft^{+} z \wedge z \triangleleft^{+} y\right]\right]
\end{aligned}
$$

Here property $P$ is meant to be the property identifying a relevant intervener for the relation meant to hold between $x$ and $y$. Note that this property could include that some other node be the left successor of $z$ with certain properties, that is, this general scheme fits cases where the intervening item is not itself directly on the path between $x$ and $y$. This formula was compiled by us and yields the automaton in Figure 3. Here the first bit position indicates membership in $P$, the second is for $x$ and the third for $y$.

$$
\begin{gathered}
\mathcal{A}=\left\langle A, \Sigma, a_{0}, F, \alpha\right\rangle, \\
A=\left\{a_{0}, a_{1}, a_{2}, a_{3}, a_{4}\right\}, \\
F=\left\{a_{3}\right\}, \\
\alpha\left(a_{0}, a_{0}, \underline{000}\right)=a_{0} \quad \alpha\left(a_{0}, a_{0}, \underline{100}\right)=a_{0} \\
\alpha\left(a_{0}, a_{0}, \underline{010}\right)=a_{2} \quad \alpha\left(a_{0}, a_{0}, \underline{110}\right)=a_{2} \\
\alpha\left(a_{0}, a_{0}, \underline{001}\right)=a_{1} \quad \alpha\left(a_{0}, a_{0}, \underline{101}\right)=a_{1} \\
\alpha\left(a_{0}, a_{1}, \underline{000}\right)=a_{1} \quad \alpha\left(a_{0}, a_{3}, \underline{000}\right)=a_{3} \\
\alpha\left(a_{0}, a_{3}, \underline{100}\right)=a_{3} \quad \alpha\left(a_{1}, a_{0}, \underline{000}\right)=a_{1} \\
\alpha\left(a_{2}, a_{1}, \underline{000}\right)=a_{3} \quad \alpha\left(a_{2}, a_{1}, \underline{100}\right)=a_{3} \\
\alpha\left(a_{3}, a_{0}, \underline{000}\right)=a_{3} \quad \alpha\left(a_{3}, a_{0}, \underline{100}\right)=a_{3} \\
\text { all other transitions are to } a_{4}
\end{gathered}
$$

Figure 3: Automaton for local c-command.

This automaton could in turn be implemented itself as Prolog code, and considered to be an optimized implementation of the given specification. Note in particular the role of the compiler as an optimizer. It outputs a minimized automaton, and the minimal automaton is a unique (up to isomorphism) definition of the given relation. Consider again the definition of AC-Command in the previous section. It is far from the most compact and elegant formula defining that relation. There exist much smaller formulas equivalent to that definition, and indeed some are suggested by the very structure of the automaton. That formula was chosen because it is an extremely straightforward formalization of the prose definition of the relation. Nonetheless, the automaton compiled from a much cleverer formalization would still be essentially the same. So no particular degree of cleverness is assumed on the part of the formalizer; optimization is done by the compiler. ${ }^{7}$

\section{MSO Logic and Constraint Logic Programming}

The automaton for a grammar formula is presumably quite a lot larger than the parse-forest automaton, that is, the automaton for the grammar conjoined with the input description. So it makes sense to search for ways to construct the parse-forest automaton which do not require the prior construction of an entire grammar automaton. In this section we consider how we might do this by by the embedding

\footnotetext{
${ }^{7}$ The structure of the formula does often have an effect on the time required by the compiler; in that sense writing MSO formalizations is still Logic Programming.
} 
of the MSO constraint language into a constraint logic programming scheme. The constraint base is an automaton which represents the incremental accumulation of knowledge about the possible valuations of variables. As discussed before, automata are a way to represent even infinite numbers of valuations with finite means, while still allowing for the efficient extraction of individual valuations. We incrementally add information to this constraint base by applying and solving clauses with their associated constraints. That is, we actually use the compiler on line as the constraint solver. Some obvious advantages include that we can still use our succinct and flexible constraint language, but gain (a) a more expressive language, since we now can include inductive definitions of relations, and (b) a way of guiding the compilation process by the specification of appropriate programs.

We define a relational extension $\mathcal{R}$ (WS2S) of our constraint language following the Höhfeld and Smolka scheme (Höhfeld and Smolka 1988). From the scheme we get a sound and complete, but now only semi-decidable, operational interpretation of a definite clause-based derivation process. The resulting structure is an extension of the underlying constraint structure with the new relations defined via fixpoints.

As usual, a definite clause is an implication with an atom as the head and a body consisting of a satisfiable MSO constraint and a (possibly empty) conjunction of atoms. A derivation step consists of two parts: goal reduction, which substitutes the body of a goal for an appropriate head, and constraint solving, which means in our case that we have to check the satisfiability of the constraint associated with the clause in conjunction with the current constraint store. For simplicity we assume a standard left-to-right, depth-first interpreter for the execution of the programs. The solution to a search branch of a program is a satisfiable constraint, represented in "solved form" as an automaton. Note that automata do make appropriate solved forms for systems of constraints: minimized automata are normal forms, and they allow for the direct and efficient recovery of particular solutions.

Intuitively, we have a language which has an operational interpretation similar to Prolog with the differences that we interpret it not on the Herbrand universe but on $\mathrm{N}_{2}$, that we use MSO constraint solving instead of unification and that we can use defined (linguistic) primitives directly.

The resulting system is only semi-decidable, due to the fact that the extension permits monadic second order variables to appear in recursively defined clauses. So if we view the inductively defined relations as part of an augmented signature, this signature contains relations on sets. These allow the specification of undecidable relations; for example, Morawietz (1997) shows how to encode the PCP. If we limit ourselves to just singleton variables in any directly or indirectly recursive clause, every relation we define stays within the capacity of MSO logic, ${ }^{8}$ since, if they are first order inductively definable, they are explicitly second order definable (Rogers 1994). Since this does not take us beyond the power of MSO logic and natural language is known not to be context-free, the extra power of $\mathcal{R}$ (WS2S) offers a way to get past the context-free boundary.

To demonstrate how we now split the work between the compiler and the CLP interpreter, we present a simple example. Consider the following naïve specification of a lexicon: ${ }^{9}$

$$
\begin{aligned}
& \operatorname{Lexicon}(x) \stackrel{\text { def }}{\Longleftrightarrow} \quad(x \in \operatorname{Sees} \wedge x \in V \wedge \ldots) \\
& \vee(x \in J \text { ohn } \wedge x \in N \wedge \ldots) \\
& \vee \quad(x \in \operatorname{Mary} \wedge x \in N \wedge \ldots)
\end{aligned}
$$

We have specified a set called Lexicon via a disjunctive specification of lexical labels, e.g. Sees, and the appropriate combination of features, e.g. $V$. Naïvely, at least, every feature we use must have its own bit position, since in the logic we treat features as set variables. So, the alphabet size with the encoding as bitstrings will be at least $2^{\mid \text {Alphabet } \mid}$. It is immediately clear that the compilation of such an automaton is extremely unattractive, if at all feasible.

We can avoid having to compile the whole lexicon by having separate clauses for each lexical entry in the CLP extension. Notational conventions will be that constraints associated with clauses are written in curly brackets and subgoals in the body are separated by \&'s. Note that relations defined in $\mathcal{R}$ (WS2S) are written lowercase.

$$
\begin{aligned}
& \text { lexicon }(x) \longleftarrow\{x \in \text { Sees } \wedge x \in V \wedge \ldots\} \\
& \text { lexicon }(x) \longleftarrow\{x \in J o h n \wedge x \in N \wedge \ldots\} \\
& \text { lexicon }(x) \longleftarrow\{x \in \operatorname{Mary} \wedge x \in N \wedge \ldots\}
\end{aligned}
$$

This shifts the burden of handling disjunctions to the interpreter. The intuitive point should be clear: it

\footnotetext{
${ }^{8}$ Relations on individuals describe sets which are expressible as monadic predicates.

${ }^{9} \mathrm{Here}$ and in the following we treat free variables as being stored in a global table so that we do not have to present them in each and every constraint. In particular, without this lexicon would have the additional arguments Sees, V, John, N, Mary and all free variables appearing in the other definitions.
} 
is not the case that every constraint in the grammar has to be expressed in one single tree automaton. We need only compile into the constraint store those which are really needed. Note that this is true even for variables appearing in the global table. In the CLP extension the appearance in the table is not coupled to the appearance in the constraint store. Only those are present in both which are part of the constraint in an applied clause.

We can also use offline compiled modules in a $\mathcal{R}$ (WS2S) parsing program. As a source of simple examples, we draw on the definitions from the lectures on P\&P parsing presented in Johnson (1995). In implementing a program such as Johnson's simplified parse relation-see Figure 4-we can in principle define any of the subgoals in the body either via precompiled automata (so they are essentially treated as facts), or else providing them with more standard definite clause definitions.

$$
\begin{array}{rlr}
\text { parse(Words, Tree }) & \longleftarrow & \\
& \{\text { Tree }(\text { Words })\} & \& \\
& \text { yield }(\text { Words }, \text { Tree }) & \& \\
& x b a r(\text { Tree }) & \& \\
& \operatorname{ecp}(\text { Tree }) &
\end{array}
$$

Figure 4: parse as in Johnson (1995)

In more detail, Words denotes a set of nodes labeled according to the input description. Our initial constraint base, which can be automatically generated from a Prolog list of input words, is the corresponding tree automaton. The associated constraint Tree is easily compilable and serves as the initialization for our parse tree. The yield and ecp predicates can easily be explicitly defined and, if practically compilable (which is certainly the case for yield), could then be treated as facts. The $x b a r$ predicate, on the other hand, is a disjunctive specification of licensing conditions depending on different features and configurations, e.g., whether we are faced with a binary-, unary- or non-branching structure, which is better expressed as several separate rules. In fact, since we want the lexicon to be represented as several definite clauses, we cannot have $x b a r$ as a simple constraint. This is due to the limitation of the constraints which appear in the definite clauses to (pure) MSO constraints.

We now have another well-defined way of using the offline compiled modules. This, at least, separates the actual processing issues (e.g., parse) from the linguistically motivated modules (e.g., ecp). One can now see that with the relational extension, we can not only use those modules which are compilable di- rectly, but also guide the compilation procedure. In effect this means interleaving the intersection of the grammar and the input description such that only the minimal amount of information to determine the parse is incrementally stored in the constraint base.

Furthermore, the language of $\mathcal{R}$ (WS2S) is sufficiently close to standard Prolog-like programming languages to allow the transfer of techniques and approaches developed in the realm of P\&P-based parsing. In other words, it needs only little effort to translate a Prolog program to a $\mathcal{R}$ (WS2S) one.

\section{Conclusions and Outlook}

In this paper we presented a first step towards the realization of a system using automata-based theoremproving techniques to implement linguistic processing and theory verification. Despite the staggering complexity bound the success of and the continuing work on these techniques in computer science promises a useable tool to test formalization of grammars. The advantages are readily apparent. The direct use of a succinct and flexible description language together with an environment to test the formalizations with the resulting finite, deterministic tree automata offers a way of combining the needs of both formalization and processing. And furthermore, the CLP extension offers an even more powerful language which allows a clear separation of processing and specification issues while retaining the power and flexibility of the original. Since it allows the control of the generation process, the addition of information to the constraint base is dependent on the input which keeps the number of variables smaller and by this the automata more compact.

Nevertheless it remains to be seen how far the system can be advanced with the use of an optimized theorem-prover. The number of variables our current prototype can handle lies between eight and eleven. ${ }^{10}$ This is not enough to compile or test all interesting aspects of a formalization. So further work will definitly involve the optimization of the prototype implementation, while we await the development of more sophisticated tools like MONA++. It seems to be promising to improve the (very basic) CLP interpreter, too. The Höhfeld and Smolka scheme allows the inclusion of existential quantification into the relational extension. We intend to use this to provide the theoretical background of the implementation of a garbage collection procedure which projects variables from the constraint store which are either local to a definite clause or

\footnotetext{
${ }^{10}$ Note that this corresponds to 256 to 2048 different bitstrings.
} 
explicitly marked for projection in the program so that the constraint store can be kept as small as possible.

\section{Acknowledgements}

This work has been supported by the project $A 8$ of the SFB 340 of the Deutsche Forschungsgemeinschaft. We wish especially to thank Uwe Mönnich and Jim Rogers for discussions and advice. Needless to say, any errors and infelicities which remain are ours alone.

\section{References}

Ayari, A., Basin, D. and Podelski, A. (1997). LISA: A specification language based on WS2S, Ms, Universität Freiburg. Submitted to CSL'97.

Basin, D. and Klarlund, N. (1995). Hardware verification using monadic second-order logic, Computer-Aided Verification (CAV' '95), LNCS 939, Springer, pp. 31-41.

Biehl, M., Klarlund, N. and Rauhe, T. (1996). Algorithms for guided tree automata, Proc. WIA ' 96 , LNCS, Springer-Verlag.

Büchi, J. R. (1960). Weak second-order arithmetic and finite automata, Zeitschrift für mathematische Logik und Grundlagen der Mathematik 6: 6692.

Doner, J. (1970). Tree acceptors and some of their applications, Journal of Computer and System Sciences 4: 406-451.

Frank, R. and Vijay-Shanker, K. (1995). Ccommand and grammatical primitives, Presentation at the 18th GLOW Colloquium. University of Tromsø.

Gécseg, F. and Steinby, M. (1984). Tree Automata, Akadémiai Kiadó, Budapest.

Henriksen, J. G., Jensen, J., Jørgensen, M., Klarlund, N., Paige, R., Rauhe, T. and Sandhol, A. (1995). MONA: Monadic second-order logic in practice, in Brinksma, Cleaveland, Larsen, Margaria and Steffen (eds), TACAS '95, LNCS 1019, Springer, pp. 89-110.

Höhfeld, M. and Smolka, G. (1988). Definite relations over constraint languages, LILOG Report 53, IBM Deutschland, Stuttgart, Germany.

Johnson, M. (1994). Two ways of formalizing grammars, Linguistics and Philosophy 17: 221-248.

Johnson, M. (1995). Constraint-based natural language parsing, ESSLLI '95, Barcelona, Course notes.
Kayne, R. S. (1994). The Antisymmetry of Syntax, MIT Press, Cambridge, Mass. and London, England.

Kelb, P., Margaria, T., Mendler, M. and Gsottberger, C. (1997). Mosel: A flexible toolset for monadic second-order logic, in E. Brinksma (ed.), TACAS '97.

Morawietz, F. (1997). Monadic second order logic, tree automata and constraint logic programming, Arbeitspapiere des SFB 340 86, SFB 340, Universität Tübingen.

Morawietz, F. and Cornell, T. L. (1997). On the recognizability of relations over a tree definable in a monadic second order tree description language, Arbeitspapiere des SFB 340 85, SFB 340, Universität Tübingen.

Niehren, J, and Podelski, A. (1992). Feature automata and recognizable sets of feature trees, in M.-C. Gaudel and J.-P. Jouannaud (eds), Proceedings of the 4th International Joint Conference on Theory and Practice of Software Development, Springer, LNCS 668, pp. 356-375.

Rabin, M. O. (1969). Decidability of second-order theories and automata on infinite trees, Transactions of the AMS 141: 1-35.

Rizzi, L. (1990). Relativized Minimality, MIT Press.

Rogers, J. (1994). Studies in the Logic of Trees with Applications to Grammar Formalisms, $\mathrm{PhD}$ thesis, University of Delaware. CS-Technical Report No. 95-04.

Rogers, J. (1996). A model-theoretic framework for theories of syntax, Proc. of the 34th Annual Meeting of the $A C L$, Santa Cruz, USA.

Thatcher, J. W. and Wright, J. B. (1968). Generalized finite automata theory with an application to a decision problem of second-order logic, Mathematical Systems Theory 2(1): 57-81.

van Noord, G. (1995). The intersection of finite state automata and definite clause grammars, Proc. of the 33th Annual Meeting of the $A C L$, Boston. 